\title{
Experimental Analyses on Parabolic Solar Collector at Various Operating Conditions
}

\author{
Rajat Sharma*, Ankur Geete \\ Mechanical Engineering Department, S. D. Bansal College of Technology, India
}

Copyright $\subset 2017$ by authors, all rights reserved. Authors agree that this article remains permanently open access under the terms of the Creative Commons Attribution License 4.0 International License

\begin{abstract}
This research work is concerned with comparative experimental analyses performed on parabolic solar collector. It presents the experimental analyses on parabolic solar collector at various operating conditions. For this experimental work, parabolic solar collector was fabricated. Various comparisons have been done between mirror concentrator and aluminium sheet concentrator. Experimental readings have been taken at 12:30 PM and at 01:30 PM and then performance of the solar collector has been found. For performance analyses, different pipe materials have been selected like copper pipe, aluminium pipe, brass pipe and mild steel pipe as receiver pipes. And different fluids have been selected for analyses like water and antifreeze ethylene glycol (coolant) as working fluids. Flowing fluids outlet temperatures, heat transfer rates and instantaneous efficiencies have been found at various operating conditions and then best operating condition for solar collector has been identified. This experimental research work can be concluded as up to $92 \%$ instantaneous efficiency and $12.2^{\circ} \mathrm{C}$ temperature difference between inlet-outlet temperatures are achieved with aluminium sheet collector but $1208.99 \mathrm{~W}$ heat transfer rate is found through mirror collector with copper pipe and coolant. After all experiments, calculations and graphs have been plotted, concluded that overall performance of fabricated solar collector with the aluminum sheet collector, copper pipe and coolant is the best.
\end{abstract}

Keywords Parabolic Solar Collector, Heat Transfer Rate, Instantaneous Efficiency, Outlet Temperature

\section{List of Nomenclatures}

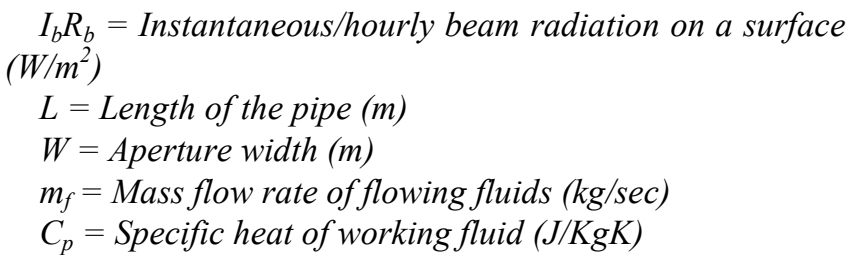

\author{
$Q_{u}=$ Rate of heat transfer $(W)$ \\ $\eta_{i b}=$ Instantaneous efficiency \\ $T_{s}=$ Surface temperature of absorber pipe $\left({ }^{\circ} \mathrm{C}\right)$ \\ $T_{f i}=$ Inlet temperature of flowing fluid in absorber pipe \\ $\left({ }^{\circ} \mathrm{C}\right)$ \\ $T_{f_{o}}=$ Outlet temperature of flowing fluid in absorber pipe \\ $\left({ }^{\circ} \mathrm{C}\right)$ \\ $f=$ focal point of parabola $(m)$ \\ $K=$ Thermal conductivity of tube material $(W-m / K)$
}

\section{Introduction}

India is a developing country, occupies $2.41 \%$ of world's land. Here the most of the population lived in villages around $70 \%$. It is too difficult to fulfill energy requirements of country. Village and remote areas presents, which do not have electricity yet. In that condition the non-conventional energy sources are the best, easy and economical. Hydro, wind and solar is the better options for energy. Solar energy is the best option according to climatic condition of India. Available all over without any cost, just need appliance to restore and use energy.

Solar energy is the major non-conventional source of energy. It is natural and free of cost without any kind of pollutants or harmful effect to the environment. Now in the $21^{\text {st }}$ century the environment condition of the earth is too affected by fossil fuels which release gases to the atmosphere, cause of that living species on earth having very big problem to live healthy and long. Also the temperature of earth increases year by year. In the modern world the most required aspect is energy. However, the energy requirements of the world fulfilled but the non-conventional source of energy are having lack of technology and difficult to store and they are not economical. Whenever the research's done with non-conventional sources makes them economical. If people not aware of it and the important steps not taken now, then the source of conventional energies will lost in few years and whole world see the earth without energy. So this is time to look forward and work for the future, next generation will be thankful to us.

Solar energy is a green energy and will facilitate reduction 
in greenhouse affects. A lot of research works have been done on it by many researchers. Kumar K. V. P. et al [2] have done experimental studies on parabolic solar collector with Sun tracking mechanism. They have worked on improving performance of parabolic solar collector with tracking mechanism at different operating conditions and they found mirror collector performed well in dry weather condition than aluminium collector. Tzivanidis C. et al [3] have simulated parabolic solar collector with commercial software Solid works which can solve complicated problems in easy way. Efficiency of the collector, heat transfer rate and convective heat transfer coefficient has also been predicted from this model. Liu X. et al [4] have investigated effect on instantaneous efficiency by multi factors in cold environment. For this investigation, they have used Sol Trace software with CFD software. Mohamad A. et al [5] worked on thermal performance of the solar collector and identified different ways of heat loss from the collector. They have also found flowing fluids, pipe and glass temperatures at various conditions.

The parabolic collector is that type of thermal collector which is bent as parabola. The bent face has polished mirrors or polished aluminium sheet for reflection of heat energy on absorber or receiver pipe placed at focal length of parabola. Source and sink connected to the receiver pipe for working fluid flow. Rate of heat transfer depends on mass flow rate, controlled by valve given at outlet of receiver pipe.

Experimental comparison in research work would be applied here because parabolic solar concentrator of mirror is tough design in single piece and if it is fitted in small parts then the performance would be affected; that's why aluminium sheet parabolic concentrator are prepared and assembled for experiments. Aluminium sheet concentrator is easy to design and assemble, also easily available everywhere. Reflectivity for mirror is 1 and for Al polished sheet is 0.96 . After that, receiver pipe materials are selected. Copper pipe is used for heat exchange device because thermal conductivity of copper is higher than aluminium, brass and mild steel. Thermal conductivity of various materials are - thermal conductivity for copper $(\mathrm{K})$ is 386 $\mathrm{Wm} / \mathrm{K}$, thermal conductivity for aluminium $(\mathrm{K})$ is 204 $\mathrm{Wm} / \mathrm{K}$, thermal conductivity for brass $(\mathrm{K})$ is $116 \mathrm{Wm} / \mathrm{K}$ and thermal conductivity for mild steel $(\mathrm{K})$ is $52 \mathrm{Wm} / \mathrm{K}$. Performance of pipe materials also depend on its properties like transmissivity and absorptivity. These terms are important because sun rays reflect through collector to receiver pipe surface in the form of radiation. Working fluid used commonly water because of higher specific heat and its availability and used repeatedly but water is corrosive and having low boiling and low freezing point. Also scale formation and its cutting fluid properties make it lower valuable as working fluid. Coolant (antifreeze ethylene glycol) is used as working fluid because it is non-corrosive, having higher boiling point and antifreeze properties. Coolant is also non sticky and low friction fluid. Specific heat of water $\left(\mathrm{C}_{\mathrm{p}}\right)$ is $4.186 \mathrm{~kJ} / \mathrm{kgK}$ and specific heat of coolant $\left(\mathrm{C}_{\mathrm{p}}\right)$ is $2.481 \mathrm{~kJ} / \mathrm{kgK}$. Layout of typical parabolic solar system is shown in figure 1. Figure 2 and figure 3 show two different solar collectors which have been used in experimental analyses.

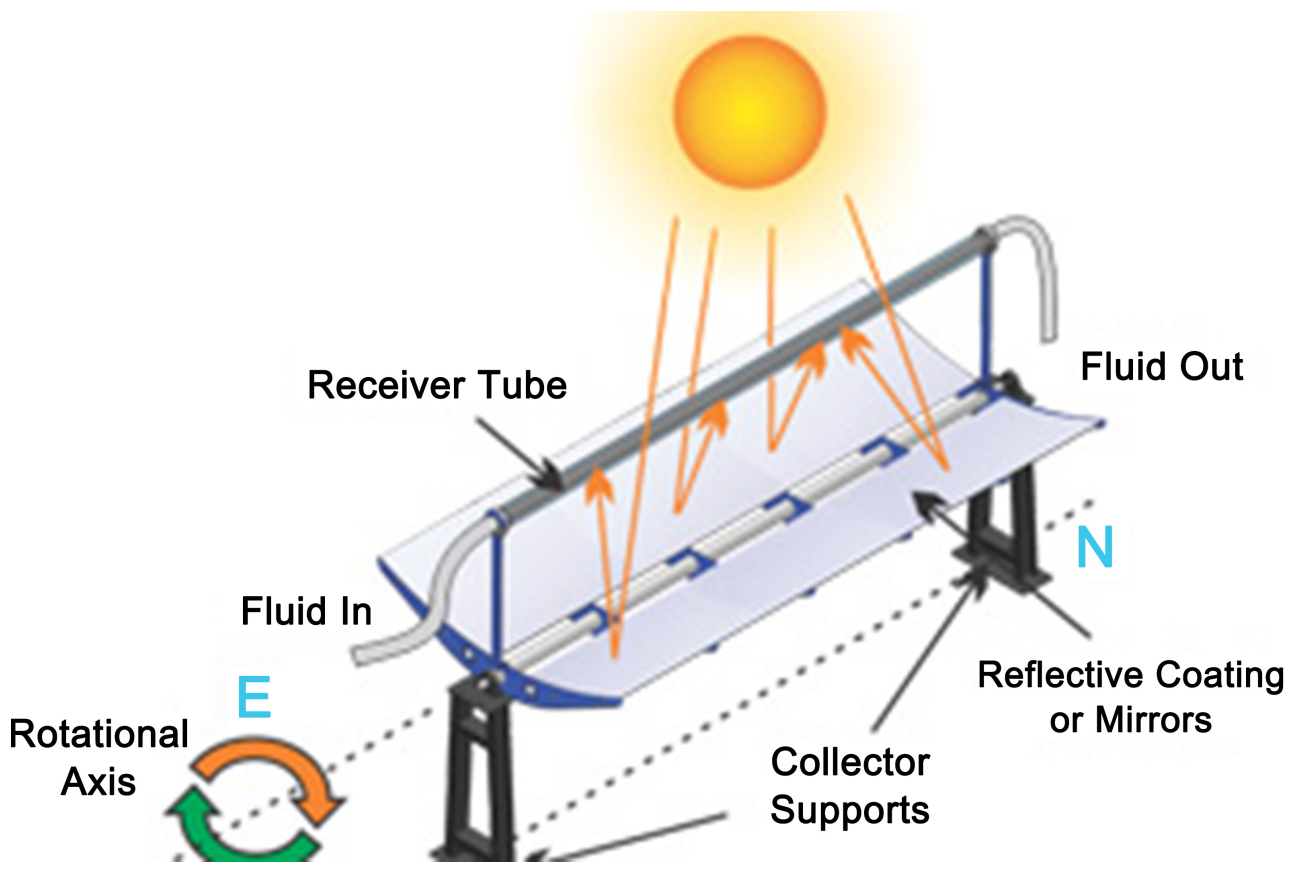

Figure 1. Layout of parabolic solar system 
Important parts of parabolic solar collector are shown in Figure 1 which is foundation supports, parabolic collector, receiver/absorber tube and rotational axis of collector.

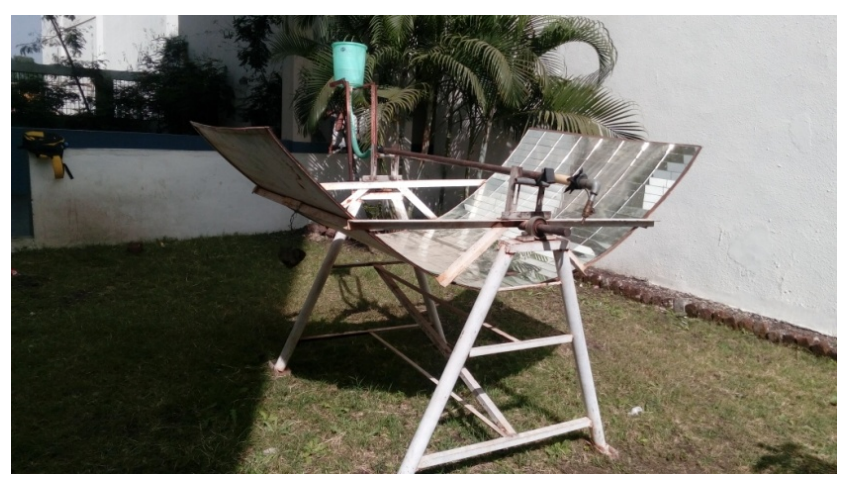

Figure 2. Fabricated parabolic solar collector with polished mirror collector

Figure 2 shows polished mirror collector and the whole arrangement of the parabolic solar collector. Here the dimensions of the collector are ( $8 \times 4$ feet) and the pieces of the mirror pasted on the collector surface are $(152.4 \times 50.8 \mathrm{x}$ $2 \mathrm{~mm}$ ). Also length of receiver tube is $1.32 \mathrm{~m}$. Bucket was used as source and fitted at some height from setup and valve is given at outlet to control flow rate.

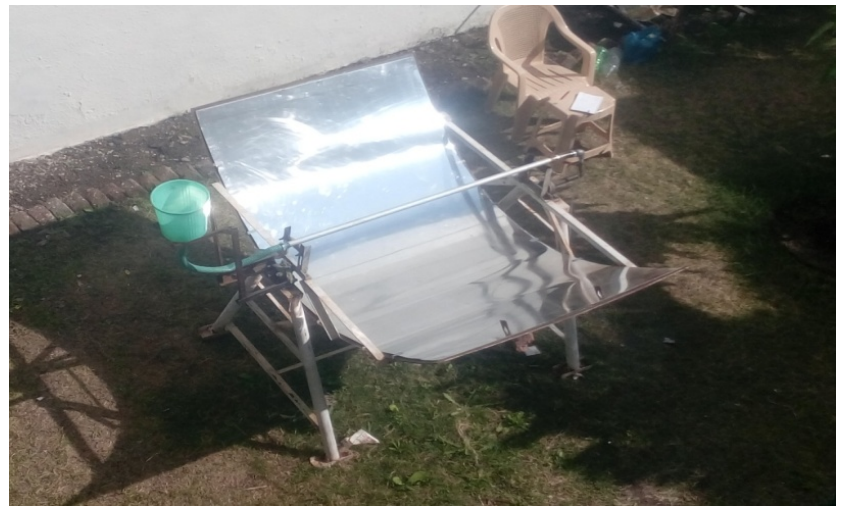

Figure 3. Fabricated parabolic solar collector with polished aluminium sheet collector

The arrangement of parabolic solar collector shows a polished aluminium sheet served as the collector in Figure 3. Here the dimensions of the aluminium sheet collector are $(8$ $x$ 4) feet and 18 gauge $(1.2 \mathrm{~mm})$ thickness. Other arrangements are same as mirror collector.

\section{Parabolic Solar Collector's Specifications are as Follows:}

(1) Collector aperture area (A) is $2.486 \mathrm{~m}^{2}$,

(2) Collector aperture width (W) is $2.04 \mathrm{~m}$,

(3) Aperture length (L) is $1.219 \mathrm{~m}$,

(4) Length of absorber pipe (1) is $1.321 \mathrm{~m}$,

(5) Inner and outer diameters of receiver pipe $\left(D_{i}\right.$ and $\left.D_{o}\right)$ $0.028 \mathrm{~m}$ and $0.032 \mathrm{~m}$ respectively,

(6) Aperture to length ratio 1.673 ,

(7) Mode of tracking North-South horizontal,
(8) Solar intensities at $12.30 \mathrm{PM}$ and $01.30 \mathrm{PM}$ is $526.90 \mathrm{~W} / \mathrm{m}^{2}$ and $529.0 \mathrm{~W} / \mathrm{m}^{2}$ respectively,

(9) Densities of water and antifreeze ethylene glycol $\left(\rho_{w}\right.$ and $\rho_{c}$ ) are $928 \mathrm{~kg} / \mathrm{m}^{3}$ and $944 \mathrm{~kg} / \mathrm{m}^{3}$ respectively and

(10) Specific heats for water and antifreeze ethylene glycol $\left(\mathrm{C}_{\mathrm{w}}\right.$ and $\left.\mathrm{C}_{\mathrm{c}}\right)$ are $4.186 \times 10^{3} \mathrm{~J} / \mathrm{kg}-\mathrm{K}$ and $2.481 \times 10^{3} \mathrm{~J} / \mathrm{kg}-\mathrm{K}$ respectively.

\section{Methodology}

Experiments have been performed with two collectors (namely made of polished mirror and Al sheet), two flowing fluids (water and antifreeze ethylene glycol) and four receiver/absorber pipes $(\mathrm{Cu}, \mathrm{Al}, \mathrm{Br}$ and $\mathrm{MS})$. All readings have been taken under $526.90 \mathrm{~W} / \mathrm{m}^{2}$ and $529.0 \mathrm{~W} / \mathrm{m}^{2}$ solar intensities at 12:30 PM and 01:30 PM respectively [1]. Entire research analyses performed in the month of November, 2016 at Sushila Devi Bansal College of Technology, Indore, Madhya Pradesh, India (Latitude - 22 $43^{\prime} 4.51^{\prime \prime} \mathrm{N}$ and Longitude $-75^{\circ} 49^{\prime} 59.88^{\prime \prime} \mathrm{E}$ ). In this research analyses, 128 readings have been taken at different operating conditions and different combinations. Experiment procedure is followed as, the source (bucket) is filled with fluid (water or coolant) continuously and it flows through receiver pipe. The flow rate is controlled by valve which is located at outlet of receiver pipe. At outlet, discharge fluid is collected in a calibrated beaker of $250 \mathrm{ml}$. Then mass flow rate of flowing fluid is calculated. Readings are taken at four different flow rates with all combinations. After finding inlet/outlet temperatures, heat transfer rates and instantaneous efficiencies have been calculated. Following relations have been used to calculate heat transfer rate and instantaneous efficiency $[1,6]$.

Useful heat gain rate,

$$
\mathrm{Q}_{\mathrm{u}}=\mathrm{m}_{\mathrm{f}} \mathrm{C}_{\mathrm{p}}\left(\mathrm{T}_{\mathrm{fo}}-\mathrm{T}_{\mathrm{fi}}\right)
$$

Instantaneous efficiency,

$$
\eta_{\mathrm{ib}}=\left(\mathrm{Q}_{\mathrm{u}}\right) /\left(\mathrm{I}_{\mathrm{b}} \mathrm{R}_{\mathrm{b}} \mathrm{W} \mathrm{L}\right)
$$

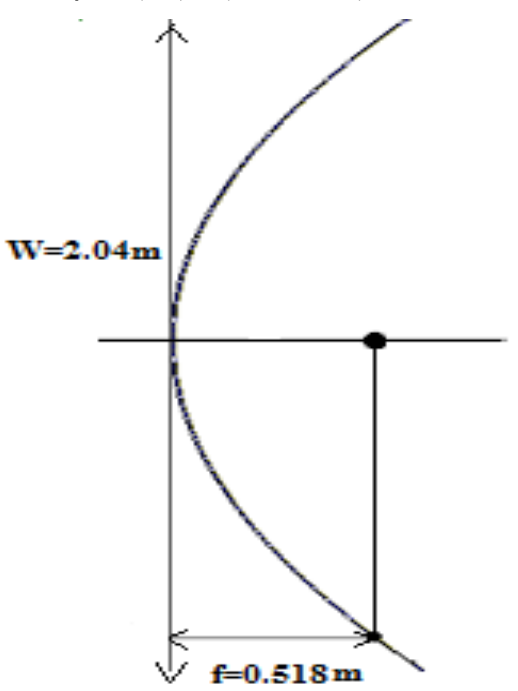

Figure 4. Graphical method to find focus point for parabolic solar collector 
Figure 4 shows the schematic design of the parabolic collector and pertinent dimensions. It is simply drawn by graphical method according to our design dimensions and then found focal point. Here, $\mathrm{W}$ is width of parabola and $\mathrm{f}$ is focal point.

\section{Results and Discussions}

By number of experiments, following observations were found with the parabolic mirror concentrator and with aluminium sheet concentrator. For the absorber tube, four different materials copper, aluminium, brass and mild steel were used and two different flowing fluids water (density $928 \mathrm{~kg} / \mathrm{m}^{3}$ ) and antifreeze ethylene glycol (density $944 \mathrm{~kg} / \mathrm{m}^{3}$ ) were chosen for experiments. Readings of mirror and $\mathrm{Al}$ sheet concentrators have been shown parallel in tables 1 to 16 and experiments have been done with both concentrators at different dates. For example, table 1 shows the results of mild steel (receiver pipe) and water combination with both concentrators at 12:30 PM on 11 November 2016 and 22 November 2016 and table 2 shows the results of mild steel (receiver pipe) and antifreeze ethylene glycol combination with both concentrators at 12:30 PM. Same procedures have been followed for 01:30 PM with both concentrators and fluids on same dates. After mild steel pipe, $\mathrm{Cu}, \mathrm{Al}$ and $\mathrm{Br}$ pipes were fitted as receiver tubes and same experimental procedures were followed repeatedly.

Table 1. 12: $30 \mathrm{pm}, \mathrm{I}_{\mathrm{b}} \mathrm{R}_{\mathrm{b}}=526.9 \mathrm{~W} / \mathrm{m}^{2}$ (Mild steel-Water)

\begin{tabular}{|c|c|c|c|c|c|c|c|c|c|c|c|c|}
\hline \multirow{2}{*}{$\begin{array}{c}\text { Concentrator/ Date } \\
\text { Sr. } \\
\text { No. }\end{array}$} & \multicolumn{6}{|c|}{$\begin{array}{c}\text { Mirror concentrator/ } \\
11 \text { Nov, Friday }\left(T_{a}=26^{\circ} \mathrm{C}\right)\end{array}$} & \multicolumn{6}{|c|}{$\begin{array}{l}\text { Aluminium sheet concentrator/ } \\
22 \text { Nov, Tuesday }\left(T_{a}=28^{\circ} \mathrm{C}\right)\end{array}$} \\
\hline & $\begin{array}{c}m_{\mathrm{f}} \\
(\mathrm{kg} / \mathrm{sec})\end{array}$ & $\begin{array}{c}\mathrm{T}_{\mathrm{s}} \\
\left({ }^{\circ} \mathrm{C}\right)\end{array}$ & $\begin{array}{r}\mathrm{T}_{\mathrm{fi}} \\
\left({ }^{\circ} \mathrm{C}\right)\end{array}$ & $\begin{array}{l}\mathrm{T}_{\mathrm{fo}} \\
\left({ }^{\circ} \mathrm{C}\right)\end{array}$ & $\begin{array}{l}Q_{u} \\
(W)\end{array}$ & $\begin{array}{c}\eta \\
(\%)\end{array}$ & $\begin{array}{c}\mathrm{m}_{\mathrm{f}} \\
(\mathrm{kg} / \mathrm{sec})\end{array}$ & $\begin{array}{c}\mathrm{T}_{\mathrm{s}} \\
\left({ }^{\circ} \mathrm{C}\right)\end{array}$ & $\begin{array}{l}\mathrm{T}_{\mathrm{fi}} \\
\left({ }^{\circ} \mathrm{C}\right)\end{array}$ & $\begin{array}{l}\mathrm{T}_{\mathrm{fo}} \\
\left({ }^{\circ} \mathrm{C}\right)\end{array}$ & $\begin{array}{l}\mathbf{Q}_{\mathrm{u}} \\
(\mathbf{W})\end{array}$ & $\begin{array}{c}\eta \\
(\%)\end{array}$ \\
\hline & 0.0229 & 46 & 30 & 41 & 1054.45 & 80.47 & 0.0249 & 42 & 29 & 38 & 938.082 & 71.59 \\
\hline & 0.0248 & 44 & 30.5 & 37 & 674.783 & 51.49 & 0.0207 & 41 & 29 & 36 & 606.551 & 25.94 \\
\hline & 0.0418 & 43 & 31 & 37 & 1049.84 & 80.12 & 0.0401 & 40 & 29.5 & 35 & 923.222 & 21.37 \\
\hline & 0.0246 & 41 & 31 & 34.5 & 360.414 & 27.50 & 0.0112 & 39 & 29.5 & 35 & 257.857 & 19.67 \\
\hline
\end{tabular}

Table 2. 12: $30 \mathrm{pm}, \mathrm{I}_{\mathrm{b}} \mathrm{R}_{\mathrm{b}}=526.9 \mathrm{~W} / \mathrm{m}^{2}$ (Mild steel-Antifreeze ethylene glycol)

\begin{tabular}{|c|c|c|c|c|c|c|c|c|c|c|c|c|}
\hline \multirow{2}{*}{$\begin{array}{c}\text { Concentrator/ Date } \\
\text { Sr. } \\
\text { No. } \\
\end{array}$} & \multicolumn{6}{|c|}{$\begin{array}{c}\text { Mirror concentrator/ } \\
11 \text { Nov, Friday }\left(T_{\mathrm{a}}=26^{\circ} \mathrm{C}\right)\end{array}$} & \multicolumn{6}{|c|}{$\begin{array}{l}\text { Aluminium sheet concentrator/ } \\
22 \text { Nov, Tuesday }\left(T_{a}=28^{\circ} \mathrm{C}\right)\end{array}$} \\
\hline & $\begin{array}{c}\mathbf{m}_{\mathrm{f}} \\
(\mathrm{kg} / \mathrm{sec})\end{array}$ & $\begin{array}{c}\mathrm{T}_{\mathrm{s}} \\
\left({ }^{\circ} \mathrm{C}\right)\end{array}$ & $\begin{array}{c}\mathbf{T}_{\text {fi }} \\
\left({ }^{\circ} \mathbf{C}\right)\end{array}$ & $\begin{array}{l}T_{\text {fo }} \\
\left({ }^{\circ} \mathrm{C}\right)\end{array}$ & $\begin{array}{c}\mathbf{Q}_{\mathrm{u}} \\
(\mathbf{W})\end{array}$ & $\begin{array}{c}\eta \\
(\%)\end{array}$ & $\begin{array}{c}\mathbf{m}_{\mathrm{f}} \\
(\mathrm{kg} / \mathrm{sec})\end{array}$ & $\begin{array}{c}\mathrm{T}_{\mathrm{s}} \\
\left({ }^{\circ} \mathrm{C}\right)\end{array}$ & $\begin{array}{l}\mathrm{T}_{\mathrm{fi}} \\
\left({ }^{\circ} \mathrm{C}\right)\end{array}$ & $\begin{array}{l}\mathrm{T}_{\mathrm{fo}} \\
\left({ }^{\circ} \mathrm{C}\right)\end{array}$ & $\begin{array}{c}\mathbf{Q}_{\mathrm{u}} \\
(\mathbf{W})\end{array}$ & $\begin{array}{c}\eta \\
(\%)\end{array}$ \\
\hline & 0.0131 & 40 & 28 & 35 & 227.507 & 17.36 & 0.0186 & 37 & 26 & 31.5 & 253.806 & 19.37 \\
\hline & 0.0180 & 39.5 & 28 & 35 & 312.606 & 23.85 & 0.0412 & 37 & 26 & 32 & 613.303 & 46.80 \\
\hline & 0.0207 & 39 & 29 & 35 & 308.140 & 23.51 & 0.0223 & 38 & 27 & 33.5 & 359.621 & 27.44 \\
\hline & 0.0208 & 38 & 29 & 36 & 361.233 & 27.56 & 0.0208 & 35 & 27 & 33 & 309.628 & 23.63 \\
\hline
\end{tabular}

Table 3. 01: 30pm, $\mathrm{I}_{b} \mathrm{R}_{\mathrm{b}}=529 \mathrm{~W} / \mathrm{m}^{2}$ (Mild steel-Antifreeze ethylene glycol)

\begin{tabular}{|c|c|c|c|c|c|c|c|c|c|c|c|c|}
\hline \multirow{2}{*}{$\begin{array}{c}\text { Concentrator/ Date } \\
\text { Sr. } \\
\text { No. } \\
\end{array}$} & \multicolumn{6}{|c|}{$\begin{array}{c}\text { Mirror concentrator/ } \\
11 \text { Nov, } \text { Friday }\left(T_{a}=28^{\circ} \mathrm{C}\right)\end{array}$} & \multicolumn{6}{|c|}{$\begin{array}{l}\text { Aluminium sheet concentrator/ } \\
22 \text { Nov, Tuesday }\left(T_{a}=28^{\circ} \mathrm{C}\right)\end{array}$} \\
\hline & $\begin{array}{c}\mathbf{m}_{\mathrm{f}} \\
(\mathrm{kg} / \mathrm{sec})\end{array}$ & $\begin{array}{l}\mathrm{T}_{\mathrm{s}} \\
\left({ }^{\circ} \mathrm{C}\right)\end{array}$ & $\begin{array}{c}\mathrm{T}_{\mathrm{fi}} \\
\left({ }^{\circ} \mathrm{C}\right)\end{array}$ & $\begin{array}{l}T_{\text {fo }} \\
\left({ }^{\circ} \mathrm{C}\right)\end{array}$ & $\begin{array}{c}\mathbf{Q}_{\mathrm{u}} \\
(\mathbf{W})\end{array}$ & $\begin{array}{c}\eta \\
(\%)\end{array}$ & $\begin{array}{c}\mathrm{m}_{\mathrm{f}} \\
(\mathrm{kg} / \mathrm{sec})\end{array}$ & $\begin{array}{c}\mathrm{T}_{\mathrm{s}} \\
\left({ }^{\circ} \mathrm{C}\right)\end{array}$ & $\begin{array}{r}T_{\mathrm{fi}} \\
\left({ }^{\circ} \mathrm{C}\right)\end{array}$ & $\begin{array}{l}\mathrm{T}_{\mathrm{fo}} \\
\left({ }^{\circ} \mathrm{C}\right)\end{array}$ & $\begin{array}{c}\mathbf{Q}_{\mathrm{u}} \\
(\mathbf{W})\end{array}$ & $\begin{array}{c}\eta \\
(\%)\end{array}$ \\
\hline & 0.0487 & 44 & 33 & 38 & 604.123 & 45.92 & 0.0213 & 44 & 31 & 40 & 475.607 & 36.15 \\
\hline & 0.0336 & 43 & 33 & 39 & 500.169 & 38.02 & 0.0328 & 43 & 31 & 39.5 & 691.702 & 52.58 \\
\hline & 0.0201 & 42 & 33 & 39 & 299.208 & 22.74 & 0.0157 & 42 & 31.5 & 39 & 292.138 & 22.20 \\
\hline & 0.0310 & 41 & 33 & 39 & 461.466 & 35.07 & 0.0254 & 40 & 31.5 & 38 & 409.613 & 31.13 \\
\hline
\end{tabular}

Table 4. 01: $30 \mathrm{pm}, \mathrm{I}_{\mathrm{b}} \mathrm{R}_{\mathrm{b}}=529 \mathrm{~W} / \mathrm{m}^{2}$ (Mild steel-Water)

\begin{tabular}{|c|c|c|c|c|c|c|c|c|c|c|c|c|}
\hline \multirow{2}{*}{$\begin{array}{c}\text { Concentrator/ Date } \\
\text { Sr. } \\
\text { No. } \\
\end{array}$} & \multicolumn{6}{|c|}{$\begin{array}{c}\text { Mirror concentrator/ } \\
11 \text { Nov, Friday }\left(T_{a}=28^{\circ} \mathrm{C}\right)\end{array}$} & \multicolumn{6}{|c|}{$\begin{array}{l}\text { Aluminium sheet concentrator/ } \\
22 \text { Nov, } T_{\text {esday }}\left(T_{a}=28^{\circ} \mathrm{C}\right)\end{array}$} \\
\hline & $\begin{array}{c}\mathrm{m}_{\mathrm{f}} \\
(\mathrm{kg} / \mathrm{sec})\end{array}$ & $\begin{array}{c}\mathrm{T}_{\mathrm{s}} \\
\left({ }^{\circ} \mathrm{C}\right)\end{array}$ & $\begin{array}{l}\mathrm{T}_{\mathrm{fi}} \\
\left({ }^{\circ} \mathrm{C}\right)\end{array}$ & $\begin{array}{l}\mathrm{T}_{\mathrm{fo}} \\
\left({ }^{\circ} \mathrm{C}\right)\end{array}$ & $\begin{array}{c}\mathbf{Q}_{\mathbf{u}} \\
(\mathbf{W})\end{array}$ & $\begin{array}{c}\eta \\
(\%)\end{array}$ & $\begin{array}{c}\mathrm{m}_{\mathrm{f}} \\
(\mathrm{kg} / \mathrm{sec})\end{array}$ & $\begin{array}{c}\mathrm{T}_{\mathrm{s}} \\
\left({ }^{\circ} \mathrm{C}\right)\end{array}$ & $\begin{array}{l}\mathrm{T}_{\mathrm{fi}} \\
\left({ }^{\circ} \mathrm{C}\right)\end{array}$ & $\begin{array}{l}\mathrm{T}_{\mathrm{fo}} \\
\left({ }^{\circ} \mathrm{C}\right)\end{array}$ & $\begin{array}{c}\mathbf{Q}_{\mathrm{u}} \\
(\mathbf{W})\end{array}$ & $\begin{array}{c}\eta \\
(\%)\end{array}$ \\
\hline & 0.0215 & 32 & 21 & 27 & 539.994 & 41.04 & 0.0197 & 37 & 29 & 33 & 329.856 & 25.07 \\
\hline & 0.0337 & 31.5 & 21.5 & 26.5 & 705.341 & 53.61 & 0.0172 & 36 & 29 & 32.5 & 251.997 & 19.15 \\
\hline & 0.0129 & 30 & 22 & 26 & 215.997 & 16.41 & 0.0211 & 35 & 30 & 32.5 & 220.812 & 16.78 \\
\hline & 0.0151 & 30 & 22 & 26 & 252.834 & 19.22 & 0.0287 & 34 & 30 & 32 & 240.276 & 18.26 \\
\hline
\end{tabular}


Table 5. $12: 30 \mathrm{pm}, \mathrm{I}_{\mathrm{b}} \mathrm{R}_{\mathrm{b}}=526.9 \mathrm{~W} / \mathrm{m}^{2}$ (Copper-Water)

\begin{tabular}{|c|c|c|c|c|c|c|c|c|c|c|c|c|}
\hline \multirow{2}{*}{$\begin{array}{c}\text { Concentrator/ Date } \\
\text { Sr. } \\
\text { No. }\end{array}$} & \multicolumn{6}{|c|}{$\begin{array}{c}\text { Mirror concentrator/ } \\
12 \text { Nov, Saturday }\left(T_{a}=28^{\circ} \mathrm{C}\right)\end{array}$} & \multicolumn{6}{|c|}{$\begin{array}{l}\text { Aluminium sheet concentrator/ } \\
17 \text { Nov, Thursday }\left(T_{a}=2^{\circ} \mathrm{C}\right)\end{array}$} \\
\hline & $\begin{array}{c}\mathrm{m}_{\mathrm{f}} \\
(\mathrm{kg} / \mathrm{sec})\end{array}$ & $\begin{array}{c}\mathrm{T}_{\mathrm{s}} \\
\left({ }^{\circ} \mathrm{C}\right)\end{array}$ & $\begin{array}{l}\mathrm{T}_{\mathrm{fi}} \\
\left({ }^{\circ} \mathrm{C}\right)\end{array}$ & $\begin{array}{l}\mathrm{T}_{\mathrm{fo}} \\
\left({ }^{\circ} \mathrm{C}\right)\end{array}$ & $\begin{array}{c}\mathbf{Q u}_{\mathrm{u}} \\
(\mathbf{W})\end{array}$ & $\begin{array}{c}\eta \\
(\%)\end{array}$ & $\begin{array}{c}\mathrm{m}_{\mathrm{f}} \\
(\mathrm{kg} / \mathrm{sec})\end{array}$ & $\begin{array}{c}\mathbf{T}_{\mathrm{s}} \\
\left({ }^{\circ} \mathrm{C}\right)\end{array}$ & $\begin{array}{c}\mathrm{T}_{\mathrm{fi}} \\
\left({ }^{\circ} \mathrm{C}\right)\end{array}$ & $\begin{array}{l}\mathrm{T}_{\mathrm{fo}} \\
\left({ }^{\circ} \mathrm{C}\right)\end{array}$ & $\begin{array}{c}\mathbf{Q u}_{\mathrm{u}} \\
(\mathbf{W})\end{array}$ & $\begin{array}{c}\eta \\
(\%)\end{array}$ \\
\hline & 0.0271 & 43 & 31 & 40 & 1020.96 & 77.92 & 0.0241 & 47 & 29 & 41.2 & 1230.77 & 93.93 \\
\hline & 0.0049 & 42 & 31 & 38 & 143.579 & 10.95 & 0.0230 & 42 & 29 & 38 & 866.50 & 66.13 \\
\hline & 0.0173 & 41 & 32 & 37 & 362.089 & 27.63 & 0.0120 & 41 & 29.5 & 38 & 426.97 & 32.58 \\
\hline & 0.0429 & 41 & 32 & 36 & 718.317 & 54.82 & 0.0270 & 40 & 29.5 & 36 & 734.643 & 56.06 \\
\hline
\end{tabular}

Table 6. $12: 30 \mathrm{pm}, \mathrm{I}_{\mathrm{b}} \mathrm{R}_{\mathrm{b}}=526.9 \mathrm{~W} / \mathrm{m}^{2}$ (Copper-Antifreeze ethylene glycol)

\begin{tabular}{|c|c|c|c|c|c|c|c|c|c|c|c|c|}
\hline \multirow{2}{*}{$\begin{array}{c}\text { Concentrator/ Date } \\
\text { Sr. } \\
\text { No. } \\
\end{array}$} & \multicolumn{6}{|c|}{$\begin{array}{c}\text { Mirror concentrator/ } \\
12 \text { Nov, Saturday }\left(T_{a}=28^{\circ} \mathrm{C}\right)\end{array}$} & \multicolumn{6}{|c|}{$\begin{array}{l}\text { Aluminium sheet concentrator/ } \\
17 \text { Nov, Thursday }\left(T_{a}=28^{\circ} \mathrm{C}\right)\end{array}$} \\
\hline & $\begin{array}{c}\mathbf{m}_{\mathrm{f}} \\
(\mathrm{kg} / \mathrm{sec})\end{array}$ & $\begin{array}{r}\mathrm{T}_{\mathrm{s}} \\
\left({ }^{\circ} \mathrm{C}\right)\end{array}$ & $\begin{array}{l}\mathrm{T}_{\mathrm{fi}} \\
\left({ }^{\circ} \mathrm{C}\right)\end{array}$ & $\begin{array}{l}\mathbf{T}_{\mathrm{fo}} \\
\left({ }^{\circ} \mathbf{C}\right)\end{array}$ & $\begin{array}{c}\mathbf{Q}_{\mathrm{u}} \\
(\mathbf{W})\end{array}$ & $\begin{array}{c}\eta \\
(\%)\end{array}$ & $\begin{array}{c}\mathbf{m}_{\mathrm{f}} \\
(\mathrm{kg} / \mathrm{sec})\end{array}$ & $\begin{array}{c}\mathrm{T}_{\mathrm{s}} \\
\left({ }^{\circ} \mathrm{C}\right)\end{array}$ & $\begin{array}{r}\mathrm{T}_{\mathrm{fi}} \\
\left({ }^{\circ} \mathrm{C}\right)\end{array}$ & $\begin{array}{l}\mathrm{T}_{\mathrm{fo}} \\
\left({ }^{\circ} \mathrm{C}\right)\end{array}$ & $\begin{array}{c}\mathbf{Q u}_{\mathrm{u}} \\
(\mathbf{W})\end{array}$ & $\begin{array}{c}\eta \\
(\%)\end{array}$ \\
\hline & 0.0565 & 40 & 29 & 34 & 700.882 & 53.49 & 0.0092 & 40 & 26 & 32 & 136.950 & 10.45 \\
\hline & 0.0149 & 40 & 29.5 & 35 & 203.318 & 15.51 & 0.0136 & 40 & 26 & 32 & 202.449 & 15.45 \\
\hline & 0.0365 & 39.5 & 30 & 35 & 452.782 & 34.55 & 0.0138 & 39 & 27 & 35 & 273.902 & 20.90 \\
\hline & 0.0229 & 39 & 30 & 35 & 284.074 & 21.68 & 0.0190 & 38 & 27 & 36 & 424.251 & 32.37 \\
\hline
\end{tabular}

Table 7. $01: 30 \mathrm{pm}, \mathrm{I}_{\mathrm{b}} \mathrm{R}_{\mathrm{b}}=529 \mathrm{~W} / \mathrm{m}^{2}$ (Copper-Antifreeze ethylene glycol)

\begin{tabular}{|c|c|c|c|c|c|c|c|c|c|c|c|c|}
\hline \multirow{2}{*}{$\begin{array}{c}\text { Concentrator/ Date } \\
\text { Sr. } \\
\text { No. } \\
\end{array}$} & \multicolumn{6}{|c|}{$\begin{array}{c}\text { Mirror concentrator/ } \\
12 \text { Nov, Saturday }\left(T_{a}=30^{\circ} \mathrm{C}\right)\end{array}$} & \multicolumn{6}{|c|}{$\begin{array}{l}\text { Aluminium sheet concentrator/ } \\
17 \text { Nov, Thursday }\left(T_{a}=29^{\circ} \mathrm{C}\right)\end{array}$} \\
\hline & $\begin{array}{c}\mathrm{m}_{\mathrm{f}} \\
(\mathrm{kg} / \mathrm{sec})\end{array}$ & $\begin{array}{c}T_{s} \\
\left({ }^{\circ} \mathrm{C}\right)\end{array}$ & $\begin{array}{r}\mathrm{T}_{\mathrm{fi}} \\
\left({ }^{\mathrm{O}} \mathrm{C}\right)\end{array}$ & $\begin{array}{l}\mathbf{T}_{\text {fo }} \\
\left({ }^{\circ} \mathbf{C}\right)\end{array}$ & $\begin{array}{c}\mathbf{Q}_{\mathrm{u}} \\
(\mathbf{W})\end{array}$ & $\begin{array}{c}\eta \\
(\%)\end{array}$ & $\begin{array}{c}\mathbf{m}_{\mathrm{f}} \\
(\mathrm{kg} / \mathrm{sec})\end{array}$ & $\begin{array}{c}\mathrm{T}_{\mathrm{s}} \\
\left({ }^{\circ} \mathrm{C}\right)\end{array}$ & $\begin{array}{l}\mathrm{T}_{\mathrm{fi}} \\
\left({ }^{\circ} \mathrm{C}\right)\end{array}$ & $\begin{array}{l}\mathbf{T}_{\mathrm{fo}} \\
\left({ }^{\circ} \mathrm{C}\right)\end{array}$ & $\begin{array}{c}\mathbf{Q}_{\mathrm{u}} \\
(\mathbf{W})\end{array}$ & $\begin{array}{c}\eta \\
(\%)\end{array}$ \\
\hline & 0.0343 & 44 & 28 & 39 & 936.081 & 71.15 & 0.0185 & 43 & 33.5 & 39 & 252.44 & 19.18 \\
\hline & 0.0284 & 44 & 28.5 & 40 & 810.294 & 61.59 & 0.0123 & 43 & 33.5 & 39 & 167.83 & 12.75 \\
\hline & 0.0109 & 42 & 29 & 39.5 & 283.950 & 21.58 & 0.0165 & 42 & 34 & 39 & 204.68 & 15.55 \\
\hline & 0.0108 & 41 & 29 & 39 & 267.948 & 20.36 & 0.0147 & 41 & 34 & 39 & $\begin{array}{c}182.35 \\
4\end{array}$ & 13.86 \\
\hline
\end{tabular}

Table 8. $01: 30 \mathrm{pm}, \mathrm{I}_{\mathrm{b}} \mathrm{R}_{\mathrm{b}}=529 \mathrm{~W} / \mathrm{m}^{2}$ (Copper-Water)

\begin{tabular}{|c|c|c|c|c|c|c|c|c|c|c|c|c|}
\hline \multirow{2}{*}{$\begin{array}{c}\text { Concentrator/ Date } \\
\text { Sr. } \\
\text { No. } \\
\end{array}$} & \multicolumn{6}{|c|}{$\begin{array}{c}\text { Mirror concentrator/ } \\
12 \text { Nov, Saturday }\left(T_{a}=30^{\circ} \mathrm{C}\right)\end{array}$} & \multicolumn{6}{|c|}{$\begin{array}{l}\text { Aluminium sheet concentrator/ } \\
17 \text { Nov, Thursday }\left(T_{a}=29^{\circ} \mathrm{C}\right)\end{array}$} \\
\hline & $\begin{array}{c}\mathbf{m}_{\mathrm{f}} \\
(\mathrm{kg} / \mathrm{sec})\end{array}$ & $\begin{array}{c}\mathbf{T}_{\mathrm{s}} \\
\left({ }^{\circ} \mathrm{C}\right)\end{array}$ & $\begin{array}{r}\mathrm{T}_{\mathrm{fi}} \\
\left({ }^{\circ} \mathrm{C}\right)\end{array}$ & $\begin{array}{l}\mathrm{T}_{\mathrm{fo}} \\
\left({ }^{\circ} \mathrm{C}\right)\end{array}$ & $\begin{array}{c}\mathbf{Q}_{\mathrm{u}} \\
(\mathbf{W})\end{array}$ & $\begin{array}{c}\eta \\
(\%)\end{array}$ & $\begin{array}{c}\mathbf{m}_{\mathrm{f}} \\
(\mathrm{kg} / \mathrm{sec})\end{array}$ & $\begin{array}{r}\mathbf{T}_{\mathrm{s}} \\
\left({ }^{\circ} \mathrm{C}\right)\end{array}$ & $\begin{array}{l}T_{\text {fi }} \\
\left({ }^{\circ} \mathrm{C}\right)\end{array}$ & $\begin{array}{l}\mathrm{T}_{\mathrm{fo}} \\
\left({ }^{\circ} \mathrm{C}\right)\end{array}$ & $\begin{array}{c}\mathbf{Q}_{\mathrm{u}} \\
(\mathbf{W})\end{array}$ & $\begin{array}{c}\eta \\
(\%)\end{array}$ \\
\hline & 0.0183 & 40 & 30 & 34 & 306.415 & 23.29 & 0.0203 & 39 & 30 & 34 & 339.903 & 25.83 \\
\hline & 0.0080 & 40 & 30 & 34 & 133.952 & 10.18 & 0.0223 & 38 & 30 & 33 & 280.043 & 21.28 \\
\hline & 0.0408 & 39 & 30 & 35 & 853.944 & 64.91 & 0.0314 & 37 & 30 & 34 & 525.761 & 39.96 \\
\hline & 0.0305 & 38 & 31 & 34 & 383.019 & 29.11 & 0.0107 & 36 & 30 & 33 & 134.370 & 10.21 \\
\hline
\end{tabular}

Table 9. $12: 30 \mathrm{pm}, \mathrm{I}_{\mathrm{b}} \mathrm{R}_{\mathrm{b}}=526.9 \mathrm{~W} / \mathrm{m}^{2}$ (Aluminium-Water)

\begin{tabular}{|c|c|c|c|c|c|c|c|c|c|c|c|c|}
\hline \multirow{2}{*}{$\begin{array}{c}\text { Concentrator/ Date } \\
\text { Sr. } \\
\text { No. } \\
\end{array}$} & \multicolumn{6}{|c|}{$\begin{array}{c}\text { Mirror concentrator/ } \\
14 \text { Nov, Monday }\left(T_{a}=27^{\circ} \mathrm{C}\right)\end{array}$} & \multicolumn{6}{|c|}{$\begin{array}{l}\text { Aluminium sheet concentrator/ } \\
18 \text { Nov, Friday }\left(T_{a}=28^{\circ} \mathrm{C}\right)\end{array}$} \\
\hline & $\begin{array}{c}\mathbf{m}_{\mathrm{f}} \\
(\mathrm{kg} / \mathrm{sec})\end{array}$ & $\begin{array}{c}\mathrm{T}_{\mathrm{s}} \\
\left({ }^{\circ} \mathrm{C}\right)\end{array}$ & $\begin{array}{c}\mathrm{T}_{\mathrm{fi}} \\
\left({ }^{\circ} \mathrm{C}\right)\end{array}$ & $\begin{array}{l}\mathbf{T}_{\mathrm{fo}} \\
\left({ }^{\circ} \mathrm{C}\right)\end{array}$ & $\begin{array}{c}\mathbf{Q}_{\mathbf{u}} \\
(\mathbf{W})\end{array}$ & $\begin{array}{c}\eta \\
(\%)\end{array}$ & $\begin{array}{c}\mathrm{m}_{\mathrm{f}} \\
(\mathrm{kg} / \mathrm{sec})\end{array}$ & $\begin{array}{c}\mathrm{T}_{\mathrm{s}} \\
\left({ }^{\circ} \mathrm{C}\right)\end{array}$ & $\begin{array}{c}\mathrm{T}_{\mathrm{fi}} \\
\left({ }^{\circ} \mathrm{C}\right)\end{array}$ & $\begin{array}{l}\mathrm{T}_{\mathrm{fo}} \\
\left({ }^{\circ} \mathrm{C}\right)\end{array}$ & $\begin{array}{c}\mathbf{Q}_{\mathbf{u}} \\
(\mathbf{W})\end{array}$ & $\begin{array}{c}\eta \\
(\%)\end{array}$ \\
\hline & 0.0217 & 39 & 25 & 34 & 817.525 & 62.39 & 0.0288 & 44 & 32 & 42 & 1205.56 & 92.00 \\
\hline & 0.0114 & 38 & 25.5 & 33.5 & 381.763 & 29.13 & 0.0219 & 43 & 32 & 40 & 733.387 & 55.97 \\
\hline & 0.0192 & 37 & 26 & 34 & 642.969 & 49.07 & 0.0134 & 42 & 32.5 & 38 & 308.508 & 23.54 \\
\hline & 0.0165 & 36 & 26 & 33 & 483.483 & 36.90 & 0.0280 & 41 & 32.5 & 37 & 527.436 & 40.25 \\
\hline
\end{tabular}


Table 10. $12: 30 \mathrm{pm}, \mathrm{I}_{\mathrm{b}} \mathrm{R}_{\mathrm{b}}=526.9 \mathrm{~W} / \mathrm{m}^{2}$ (Aluminium-Antifreeze ethylene glycol)

\begin{tabular}{|c|c|c|c|c|c|c|c|c|c|c|c|c|}
\hline \multirow{2}{*}{$\begin{array}{c}\text { Concentrator/ Date } \\
\text { Sr. } \\
\text { No. } \\
\end{array}$} & \multicolumn{6}{|c|}{$\begin{array}{c}\text { Mirror concentrator } / \\
14 \text { Nov, Monday }\left(T_{\mathrm{a}}=27^{\circ} \mathrm{C}\right)\end{array}$} & \multicolumn{6}{|c|}{$\begin{array}{l}\text { Aluminium sheet concentrator/ } \\
18 \text { Nov, Friday }\left(T_{a}=28^{\circ} \mathrm{C}\right)\end{array}$} \\
\hline & $\begin{array}{c}\mathrm{m}_{\mathrm{f}} \\
(\mathrm{kg} / \mathrm{sec})\end{array}$ & $\begin{array}{c}\mathrm{T}_{\mathrm{s}} \\
\left({ }^{\circ} \mathrm{C}\right)\end{array}$ & $\begin{array}{l}\mathbf{T}_{\mathrm{fi}} \\
\left({ }^{\circ} \mathrm{C}\right)\end{array}$ & $\begin{array}{l}\mathbf{T}_{\mathrm{fo}} \\
\left({ }^{\circ} \mathrm{C}\right)\end{array}$ & $\begin{array}{l}\mathbf{Q}_{\mathrm{u}} \\
(\mathbf{W})\end{array}$ & $\begin{array}{c}\eta \\
(\%)\end{array}$ & $\begin{array}{c}\mathrm{m}_{\mathrm{f}} \\
(\mathrm{kg} / \mathrm{sec})\end{array}$ & $\begin{array}{r}T_{s} \\
\left({ }^{\circ} \mathrm{C}\right)\end{array}$ & $\begin{array}{r}\mathrm{T}_{\mathrm{fi}} \\
\left({ }^{\circ} \mathrm{C}\right)\end{array}$ & $\begin{array}{l}\mathbf{T}_{\mathrm{fo}} \\
\left({ }^{\circ} \mathrm{C}\right)\end{array}$ & $\begin{array}{l}\mathbf{Q}_{\mathrm{u}} \\
(\mathbf{W})\end{array}$ & $\begin{array}{c}\eta \\
(\%)\end{array}$ \\
\hline & 0.0332 & 34 & 25 & 30 & 411.846 & 31.43 & 0.0192 & 37 & 25 & 31 & 285.811 & 21.81 \\
\hline & 0.0182 & 33.5 & 25.5 & 29.5 & 180.616 & 13.78 & 0.0144 & 36 & 25 & 31 & 214.358 & 16.35 \\
\hline & 0.0312 & 33 & 26 & 31 & 387.036 & 29.53 & 0.0087 & 35 & 26 & 33 & 151.092 & 11.53 \\
\hline & 0.0276 & 32.5 & 26 & 30.5 & 308.140 & 23.51 & 0.0167 & 34 & 26 & 32.5 & 269.312 & 20.55 \\
\hline
\end{tabular}

Table 11. 01: $30 \mathrm{pm}, \mathrm{I}_{\mathrm{b}} \mathrm{R}_{\mathrm{b}}=529 \mathrm{~W} / \mathrm{m}^{2}$ (Aluminium-Antifreeze ethylene glycol)

\begin{tabular}{|c|c|c|c|c|c|c|c|c|c|c|c|c|}
\hline Concentrator/ Date & \multicolumn{4}{|c|}{ Mirror concentrator/ } & \multicolumn{4}{c|}{ Aluminium sheet concentrator/ } \\
\hline $\begin{array}{c}\text { Sr. } \\
\text { No. }\end{array}$ & $\begin{array}{c}\mathbf{m}_{\mathbf{f}} \\
(\mathbf{k g} / \mathbf{s e c})\end{array}$ & $\begin{array}{c}\mathbf{T}_{\mathbf{s}} \\
\left({ }^{\circ} \mathbf{C}\right)\end{array}$ & $\begin{array}{c}\mathbf{T}_{\mathbf{f i}} \\
\left({ }^{\circ} \mathbf{C}\right)\end{array}$ & $\begin{array}{c}\mathbf{T}_{\mathbf{f o}} \\
\left({ }^{\circ} \mathbf{C}\right)\end{array}$ & $\begin{array}{c}\mathbf{Q}_{\mathbf{u}} \\
(\mathbf{W})\end{array}$ & $\begin{array}{c}\mathbf{\eta} \\
(\mathbf{\%})\end{array}$ & $\begin{array}{c}\mathbf{m}_{\mathbf{f}} \\
(\mathbf{k g} / \mathbf{s e c})\end{array}$ & $\begin{array}{c}\mathbf{T}_{\mathbf{s}} \\
\left({ }^{\circ} \mathbf{C}\right)\end{array}$ & $\begin{array}{c}\mathbf{T}_{\mathbf{f i}} \\
\left({ }^{\circ} \mathbf{C}\right)\end{array}$ & $\begin{array}{c}\mathbf{T}_{\mathbf{f}} \\
\left({ }^{\circ} \mathbf{C}\right)\end{array}$ & $\begin{array}{c}\mathbf{Q}_{\mathbf{u}} \\
(\mathbf{W})\end{array}$ & $\begin{array}{c}\mathbf{\eta} \\
(\mathbf{\%})\end{array}$ \\
\hline & 0.0191 & 41 & 30 & 37 & 331.709 & 25.21 & 0.0174 & 42 & 31.5 & 37 & 237.431 & 18.04 \\
\hline & 0.0278 & 40 & 30 & 36.5 & 448.316 & 34.07 & 0.0206 & 41 & 31.5 & 36 & 229.988 & 17.48 \\
\hline & 0.0291 & 39 & 31 & 37 & 433.182 & 32.92 & 0.0303 & 39 & 32 & 36 & 300.697 & 22.85 \\
\hline & 0.0383 & 39 & 31 & 36 & 475.111 & 36.11 & 0.0196 & 38 & 32 & 36 & 194.510 & 14.78 \\
\hline
\end{tabular}

Table 12. 01: $30 \mathrm{pm}, \mathrm{I}_{\mathrm{b}} \mathrm{R}_{\mathrm{b}}=529 \mathrm{~W} / \mathrm{m}^{2}$ (Aluminium-Water)

\begin{tabular}{|c|c|c|c|c|c|c|c|c|c|c|c|c|}
\hline \multirow{2}{*}{$\begin{array}{c}\text { Concentrator/ Date } \\
\text { Sr. } \\
\text { No. } \\
\end{array}$} & \multicolumn{6}{|c|}{$\begin{array}{c}\text { Mirror concentrator/ } \\
14 \text { Nov, Monday }\left(T_{a}=30^{\circ} \mathrm{C}\right)\end{array}$} & \multicolumn{6}{|c|}{$\begin{array}{l}\text { Aluminium sheet concentrator/ } \\
18 \text { Nov, Friday }\left(T_{a}=28^{\circ} \mathrm{C}\right)\end{array}$} \\
\hline & $\begin{array}{c}\mathbf{m}_{\mathrm{f}} \\
(\mathrm{kg} / \mathrm{sec})\end{array}$ & $\begin{array}{c}\mathbf{T}_{\mathrm{s}} \\
\left({ }^{\circ} \mathrm{C}\right)\end{array}$ & $\begin{array}{l}\mathrm{T}_{\mathrm{fi}} \\
\left({ }^{\circ} \mathrm{C}\right)\end{array}$ & $\begin{array}{l}\mathrm{T}_{\text {fo }} \\
\left({ }^{\circ} \mathrm{C}\right)\end{array}$ & $\begin{array}{c}\mathbf{Q}_{\mathrm{u}} \\
(\mathbf{W})\end{array}$ & $\begin{array}{c}\eta \\
(\%)\end{array}$ & $\begin{array}{c}m_{\mathrm{f}} \\
(\mathrm{kg} / \mathrm{sec})\end{array}$ & $\begin{array}{c}\mathrm{T}_{\mathrm{s}} \\
\left({ }^{\circ} \mathrm{C}\right)\end{array}$ & $\begin{array}{l}\mathrm{T}_{\mathrm{fi}} \\
\left({ }^{\circ} \mathrm{C}\right)\end{array}$ & $\begin{array}{l}T_{\text {fo }} \\
\left({ }^{\circ} \mathrm{C}\right)\end{array}$ & $\begin{array}{c}\mathbf{Q}_{\mathrm{u}} \\
(\mathbf{W})\end{array}$ & $\begin{array}{c}\eta \\
(\%)\end{array}$ \\
\hline & 0.0227 & 33 & 23 & 29 & 570.1332 & 43.33 & 0.0210 & 37 & 29 & 32.5 & 307.671 & 23.38 \\
\hline & 0.0144 & 33 & 24 & 27 & 180.8352 & 13.74 & 0.0119 & 36 & 29 & 32 & 149.440 & 11.35 \\
\hline & 0.0337 & 32 & 24 & 28.5 & 634.8069 & 48.25 & 0.0295 & 35 & 30 & 33 & 370.461 & 28.16 \\
\hline & 0.0106 & 31 & 24 & 28 & 177.4864 & 13.49 & 0.0346 & 35 & 30 & 33 & 434.506 & 33.02 \\
\hline
\end{tabular}

Table 13. $12: 30 \mathrm{pm}, \mathrm{I}_{\mathrm{b}} \mathrm{R}_{\mathrm{b}}=526.9 \mathrm{~W} / \mathrm{m}^{2}$ (Brass-Water)

\begin{tabular}{|c|c|c|c|c|c|c|c|c|c|c|c|c|}
\hline \multirow{2}{*}{$\begin{array}{c}\text { Concentrator/ Date } \\
\text { Sr. } \\
\text { No. } \\
\end{array}$} & \multicolumn{6}{|c|}{$\begin{array}{c}\text { Mirror concentrator/ } \\
15 \text { Nov, } \text { Tuesday }\left(T_{a}=27^{\circ} \mathrm{C}\right)\end{array}$} & \multicolumn{6}{|c|}{$\begin{array}{l}\text { Aluminium sheet concentrator/ } \\
21 \text { Nov, Monday }\left(T_{a}=30^{\circ} \mathrm{C}\right)\end{array}$} \\
\hline & $\begin{array}{c}\mathbf{m}_{\mathrm{f}} \\
(\mathrm{kg} / \mathrm{sec})\end{array}$ & $\begin{array}{c}\mathrm{T}_{\mathrm{s}} \\
\left({ }^{\circ} \mathrm{C}\right)\end{array}$ & $\begin{array}{l}\mathbf{T}_{\mathrm{fi}} \\
\left({ }^{\circ} \mathrm{C}\right)\end{array}$ & $\begin{array}{l}\mathrm{T}_{\mathrm{fo}} \\
\left({ }^{\circ} \mathrm{C}\right)\end{array}$ & $\begin{array}{l}\mathbf{Q}_{\mathrm{u}} \\
(\mathbf{W})\end{array}$ & $\begin{array}{c}\eta \\
(\%)\end{array}$ & $\begin{array}{c}\mathrm{m}_{\mathrm{f}} \\
(\mathrm{kg} / \mathrm{sec})\end{array}$ & $\begin{array}{r}\mathrm{T}_{\mathrm{s}} \\
\left({ }^{\circ} \mathrm{C}\right)\end{array}$ & $\begin{array}{l}\mathrm{T}_{\mathrm{fi}} \\
\left({ }^{\circ} \mathrm{C}\right)\end{array}$ & $\begin{array}{l}\mathrm{T}_{\mathrm{fo}} \\
\left({ }^{\circ} \mathrm{C}\right)\end{array}$ & $\begin{array}{c}\mathbf{Q}_{\mathrm{u}} \\
(\mathbf{W})\end{array}$ & $\begin{array}{c}\eta \\
(\%)\end{array}$ \\
\hline & 0.0158 & 44 & 31 & 40 & 595.249 & 45.42 & 0.0112 & 44 & 29 & 37.5 & 398.507 & 30.41 \\
\hline & 0.0121 & 44 & 31 & 41.5 & 531.831 & 40.59 & 0.0215 & 43 & 29 & 39 & 899.990 & 68.68 \\
\hline & 0.0203 & 43.5 & 32 & 38.5 & 552.342 & 42.15 & 0.0182 & 41 & 30 & 36.5 & 495.203 & 37.79 \\
\hline & 0.0319 & 43 & 32 & 37 & 667.667 & 50.95 & 0.0223 & 39 & 30 & 36 & 560.086 & 42.74 \\
\hline
\end{tabular}

Table 14. 12: $30 \mathrm{pm}, \mathrm{I}_{\mathrm{b}} \mathrm{R}_{\mathrm{b}}=526.9 \mathrm{~W} / \mathrm{m}^{2}$ (Brass-Antifreeze ethylene glycol)

\begin{tabular}{|c|c|c|c|c|c|c|c|c|c|c|c|c|}
\hline \multirow{2}{*}{$\begin{array}{c}\text { Concentrator/ Date } \\
\text { Sr. } \\
\text { No. } \\
\end{array}$} & \multicolumn{6}{|c|}{$\begin{array}{c}\text { Mirror concentrator/ } \\
15 \text { Nov, } \text { Tuesday }\left(T_{a}=27^{\circ} \mathrm{C}\right)\end{array}$} & \multicolumn{6}{|c|}{$\begin{array}{l}\text { Aluminium sheet concentrator/ } \\
21 \text { Nov, Monday }\left(T_{a}=30^{\circ} \mathrm{C}\right)\end{array}$} \\
\hline & $\begin{array}{c}\mathrm{m}_{\mathrm{f}} \\
(\mathrm{kg} / \mathrm{sec})\end{array}$ & $\begin{array}{c}\mathrm{T}_{\mathrm{s}} \\
\left({ }^{\circ} \mathrm{C}\right)\end{array}$ & $\begin{array}{l}\mathrm{T}_{\mathrm{fi}} \\
\left({ }^{\circ} \mathrm{C}\right)\end{array}$ & $\begin{array}{l}\mathrm{T}_{\mathrm{fo}} \\
\left({ }^{\circ} \mathrm{C}\right)\end{array}$ & $\begin{array}{c}\mathbf{Q}_{\mathrm{u}} \\
(\mathbf{W})\end{array}$ & $\begin{array}{c}\eta \\
(\%)\end{array}$ & $\begin{array}{c}\mathbf{m}_{\mathrm{f}} \\
(\mathrm{kg} / \mathrm{sec})\end{array}$ & $\begin{array}{l}\mathrm{T}_{\mathrm{s}} \\
\left({ }^{\circ} \mathrm{C}\right)\end{array}$ & $\begin{array}{r}\mathrm{T}_{\mathrm{fi}} \\
\left({ }^{\circ} \mathrm{C}\right)\end{array}$ & $\begin{array}{l}\mathbf{T}_{\mathrm{fo}} \\
\left({ }^{\circ} \mathbf{C}\right)\end{array}$ & $\begin{array}{c}\mathbf{Q u}_{\mathrm{u}} \\
(\mathbf{W})\end{array}$ & $\begin{array}{c}\eta \\
(\%)\end{array}$ \\
\hline & 0.0206 & 38 & 29 & 34 & 255.543 & 19.50 & 0.0301 & 38 & 25 & 32 & 522.746 & 39.89 \\
\hline & 0.0198 & 38.5 & 29.5 & 34 & 221.057 & 16.87 & 0.0118 & 38.5 & 25 & 32 & 204.930 & 15.64 \\
\hline & 0.0233 & 37 & 30 & 34 & 231.229 & 17.64 & 0.0200 & 37 & 26 & 33 & 347.340 & 26.50 \\
\hline & 0.0184 & 36 & 30 & 34 & 182.601 & 13.93 & 0.0271 & 36 & 26 & 33 & 470.645 & 35.91 \\
\hline
\end{tabular}


Table 15. 01: $30 \mathrm{pm}, \mathrm{I}_{\mathrm{b}} \mathrm{R}_{\mathrm{b}}=529 \mathrm{~W} / \mathrm{m}^{2}$ (Brass-Antifreeze ethylene glycol)

\begin{tabular}{|c|c|c|c|c|c|c|c|c|c|c|c|c|}
\hline Concentrator/ Date & \multicolumn{6}{|c|}{$\begin{array}{c}\text { Mirror concentrator/ } \\
15 \text { Nov, } \text { Tuesday }\left(T_{\mathrm{a}}=30^{\circ} \mathrm{C}\right)\end{array}$} & \multicolumn{6}{|c|}{$\begin{array}{l}\text { Aluminium sheet concentrator/ } \\
21 \text { Nov, Monday }\left(T_{a}=28^{\circ} \mathrm{C}\right)\end{array}$} \\
\hline $\begin{array}{l}\text { Sr. } \\
\text { No. }\end{array}$ & $\begin{array}{c}\mathbf{m}_{\mathrm{f}} \\
(\mathrm{kg} / \mathrm{sec})\end{array}$ & $\begin{array}{c}\mathbf{T}_{\mathrm{s}} \\
\left({ }^{\circ} \mathrm{C}\right)\end{array}$ & $\begin{array}{l}\mathrm{T}_{\mathrm{fi}} \\
\left({ }^{\circ} \mathrm{C}\right)\end{array}$ & $\begin{array}{l}\mathrm{T}_{\mathrm{fo}} \\
\left({ }^{\circ} \mathrm{C}\right)\end{array}$ & $\begin{array}{l}\mathbf{Q}_{\mathrm{u}} \\
(\mathbf{W})\end{array}$ & $\begin{array}{c}\eta \\
(\%)\end{array}$ & $\begin{array}{c}\mathrm{m}_{\mathrm{f}} \\
(\mathrm{kg} / \mathrm{sec})\end{array}$ & $\begin{array}{c}\mathbf{T}_{\mathrm{s}} \\
\left({ }^{\circ} \mathrm{C}\right)\end{array}$ & $\begin{array}{r}\mathrm{T}_{\mathrm{fi}} \\
\left({ }^{\circ} \mathrm{C}\right)\end{array}$ & $\begin{array}{l}\mathrm{T}_{\mathrm{fo}} \\
\left({ }^{\circ} \mathrm{C}\right)\end{array}$ & $\begin{array}{c}\mathbf{Q}_{\mathbf{u}} \\
(\mathbf{W})\end{array}$ & $\begin{array}{c}\eta \\
(\%)\end{array}$ \\
\hline & 0.0138 & 42 & 33 & 39 & 205.426 & 15.61 & 0.0317 & 43 & 31 & 39 & 629.181 & 47.82 \\
\hline & 0.0107 & 42 & 33 & 38.5 & 146.006 & 11.09 & 0.0287 & 40 & 31 & 37 & 427.228 & 32.47 \\
\hline & 0.0139 & 41.5 & 33 & 38 & 172.429 & 13.10 & 0.0238 & 39 & 32 & 36.5 & 265.715 & 20.19 \\
\hline & 0.0240 & 41.5 & 33 & 38 & 297.720 & 22.63 & 0.0187 & 37 & 32 & 36 & 182.602 & 13.88 \\
\hline
\end{tabular}

Table 16. $01: 30 \mathrm{pm}, \mathrm{I}_{\mathrm{b}} \mathrm{R}_{\mathrm{b}}=529 \mathrm{~W} / \mathrm{m}^{2}$ (Brass-Water)

\begin{tabular}{|c|c|c|c|c|c|c|c|c|c|c|c|c|}
\hline \multirow{2}{*}{$\begin{array}{c}\text { Concentrator/ Date } \\
\text { Sr. } \\
\text { No. } \\
\end{array}$} & \multicolumn{6}{|c|}{$\begin{array}{c}\text { Mirror concentrator/ } \\
15 \text { Nov, Tuesday }\left(T_{a}=30^{\circ} \mathrm{C}\right)\end{array}$} & \multicolumn{6}{|c|}{$\begin{array}{l}\text { Aluminium sheet concentrator/ } \\
21 \text { Nov, Monday }\left(T_{a}=28^{\circ} \mathrm{C}\right)\end{array}$} \\
\hline & $\begin{array}{c}\mathrm{m}_{\mathrm{f}} \\
(\mathrm{kg} / \mathrm{sec})\end{array}$ & $\begin{array}{c}\mathrm{T}_{\mathrm{s}} \\
\left({ }^{\circ} \mathrm{C}\right)\end{array}$ & $\begin{array}{r}\mathrm{T}_{\mathrm{fi}} \\
\left({ }^{\circ} \mathrm{C}\right)\end{array}$ & $\begin{array}{l}\mathbf{T}_{\mathrm{fo}} \\
\left({ }^{\circ} \mathrm{C}\right)\end{array}$ & $\begin{array}{c}\mathbf{Q}_{\mathrm{u}} \\
(\mathbf{W})\end{array}$ & $\begin{array}{c}\eta \\
(\%)\end{array}$ & $\begin{array}{c}\mathrm{m}_{\mathrm{f}} \\
(\mathrm{kg} / \mathrm{sec})\end{array}$ & $\begin{array}{c}\mathrm{T}_{\mathrm{s}} \\
\left({ }^{\circ} \mathrm{C}\right)\end{array}$ & $\begin{array}{l}\mathrm{T}_{\mathrm{fi}} \\
\left({ }^{\circ} \mathrm{C}\right)\end{array}$ & $\begin{array}{l}\mathrm{T}_{\mathrm{fo}} \\
\left({ }^{\circ} \mathrm{C}\right)\end{array}$ & $\begin{array}{c}\mathbf{Q}_{\mathrm{u}} \\
(\mathbf{W})\end{array}$ & $\begin{array}{c}\eta \\
(\%)\end{array}$ \\
\hline & 0.0279 & 38 & 29 & 34 & 583.947 & 44.38 & 0.0339 & 36 & 29 & 32 & 425.716 & 32.36 \\
\hline & 0.0138 & 38 & 29 & 33.5 & 259.950 & 19.76 & 0.0195 & 35.5 & 29 & 32 & 244.881 & 18.61 \\
\hline & 0.0323 & 37.5 & 30 & 34 & 540.831 & 41.11 & 0.0214 & 35 & 29.5 & 32 & 223.951 & 17.02 \\
\hline & 0.0148 & 37.5 & 30 & 34 & 247.811 & 18.837 & 0.0156 & 35 & 29.5 & 32 & 163.254 & 12.41 \\
\hline
\end{tabular}

Some comparative results of heat transfer rates (HTRs) have also been shown in Figure 5 to 9. Figure 5 shows comparison of percentages of HTRs between mirror concentrator and aluminium sheet concentrator. Figure 6 shows comparison of percentages of HTRs between copper, aluminium, brass and mild steel materials with mirror concentrator. Figure 7 shows comparison of percentages of HTRs between copper, aluminium, brass and mild steel materials with aluminium sheet concentrator. Figure 8 shows comparison of percentages of HTRs between water and coolant as working fluids through receiver pipe with mirror concentrator. And, Figure 9 shows comparison of percentages of HTRs between water and coolant with aluminium concentrator.

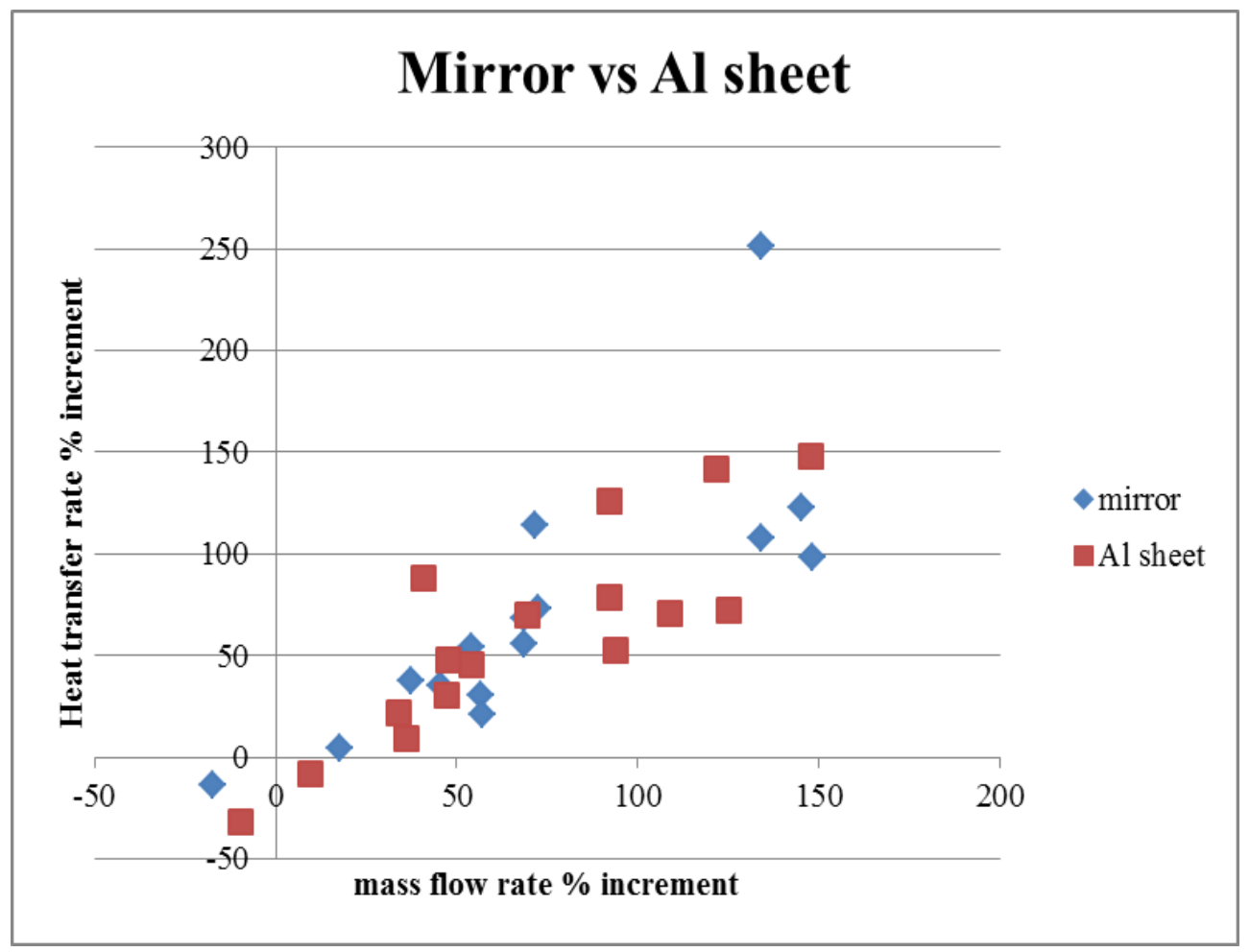

Figure 5. Graph plotted between $\%$ increment of mass flow rate and $\%$ increment in heat transfer rate for mirror and aluminium collector 


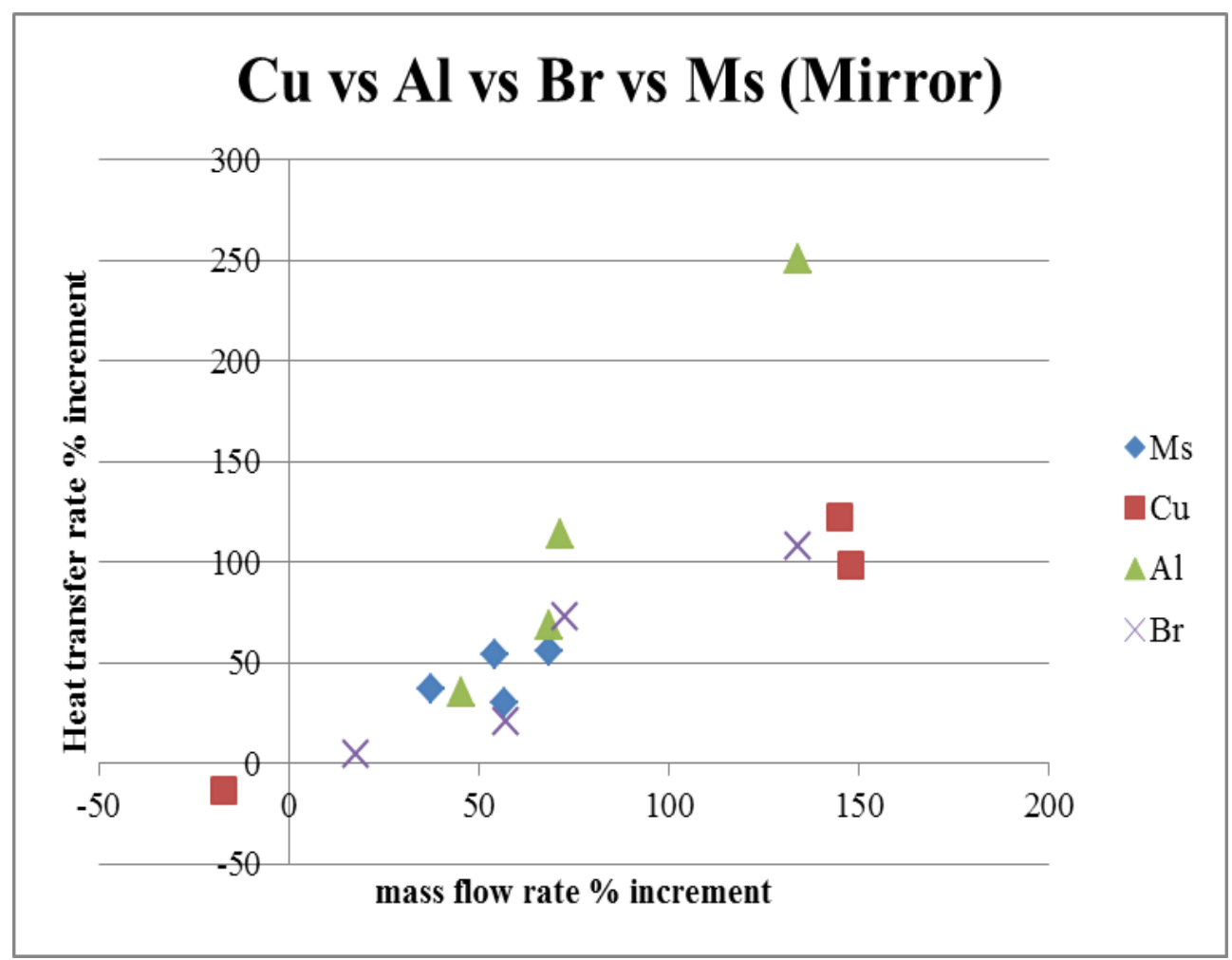

Figure 6. Graph plotted between $\%$ increment of mass flow rate and $\%$ increment in heat transfer rate for various materials with mirror collector

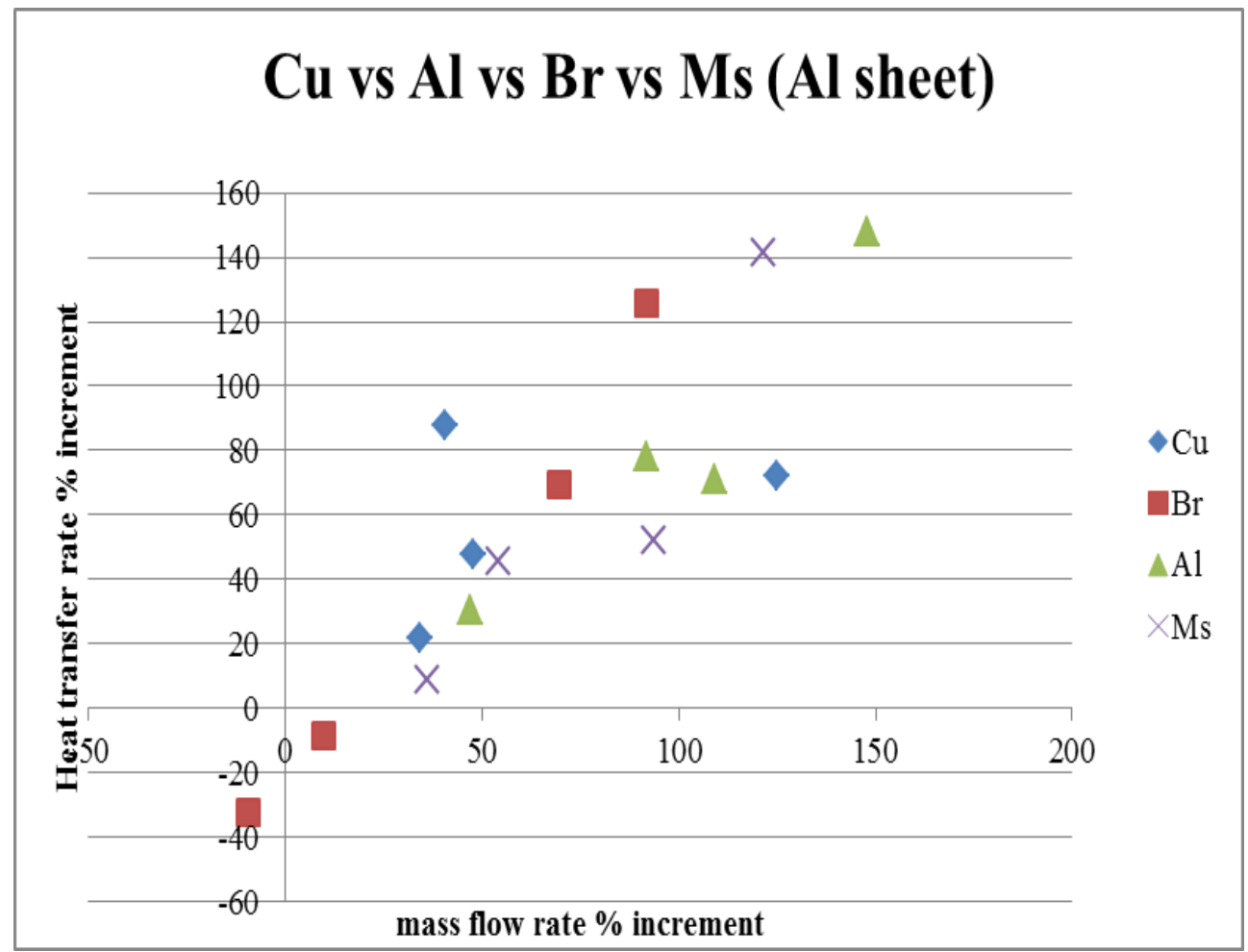

Figure 7. Graph plotted between $\%$ increment of mass flow rate and $\%$ increment in heat transfer rate for various materials with aluminium sheet collector 


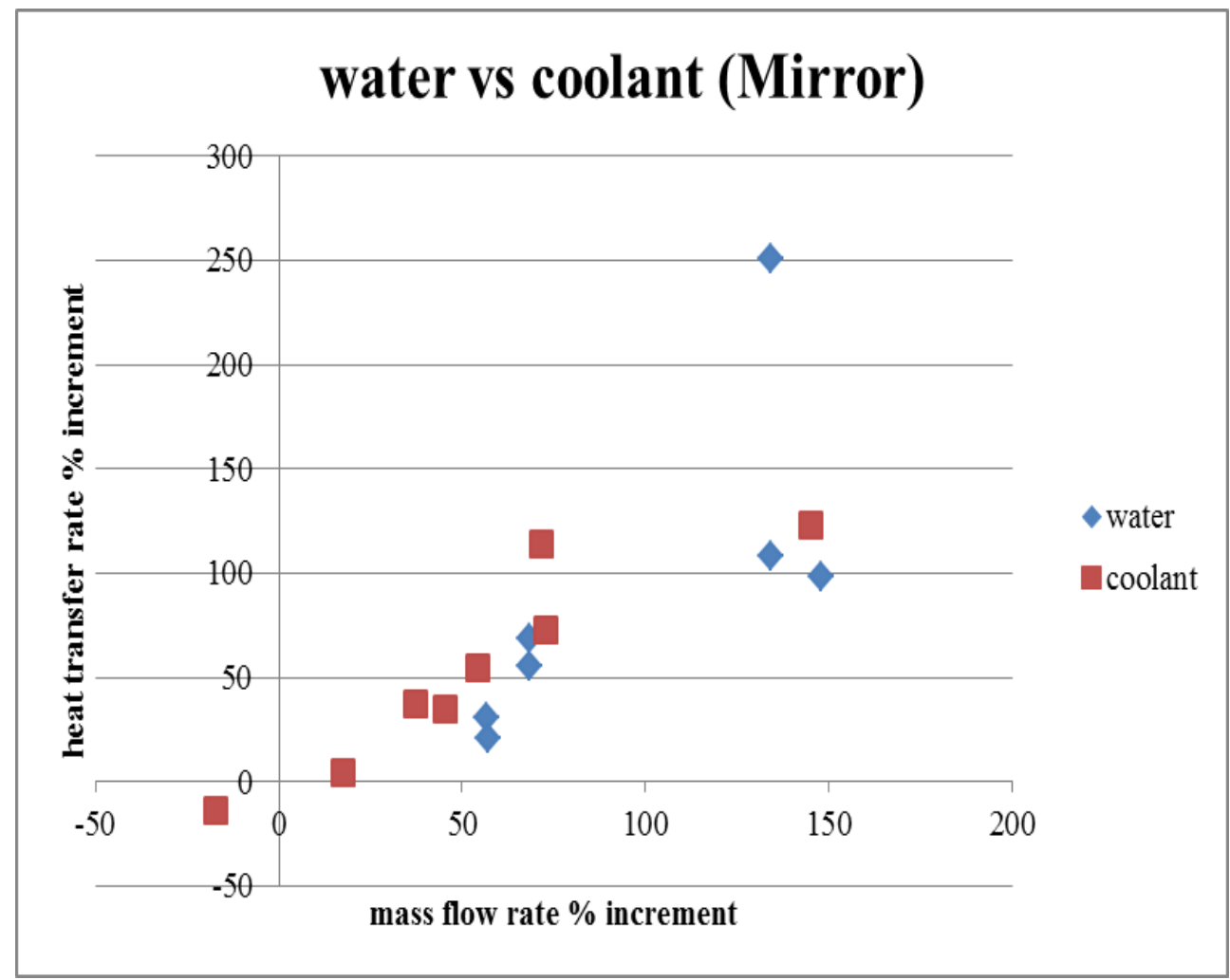

Figure 8. Graph plotted between $\%$ increment of mass flow rate and $\%$ increment in heat transfer rate with various flowing fluids for mirror collector

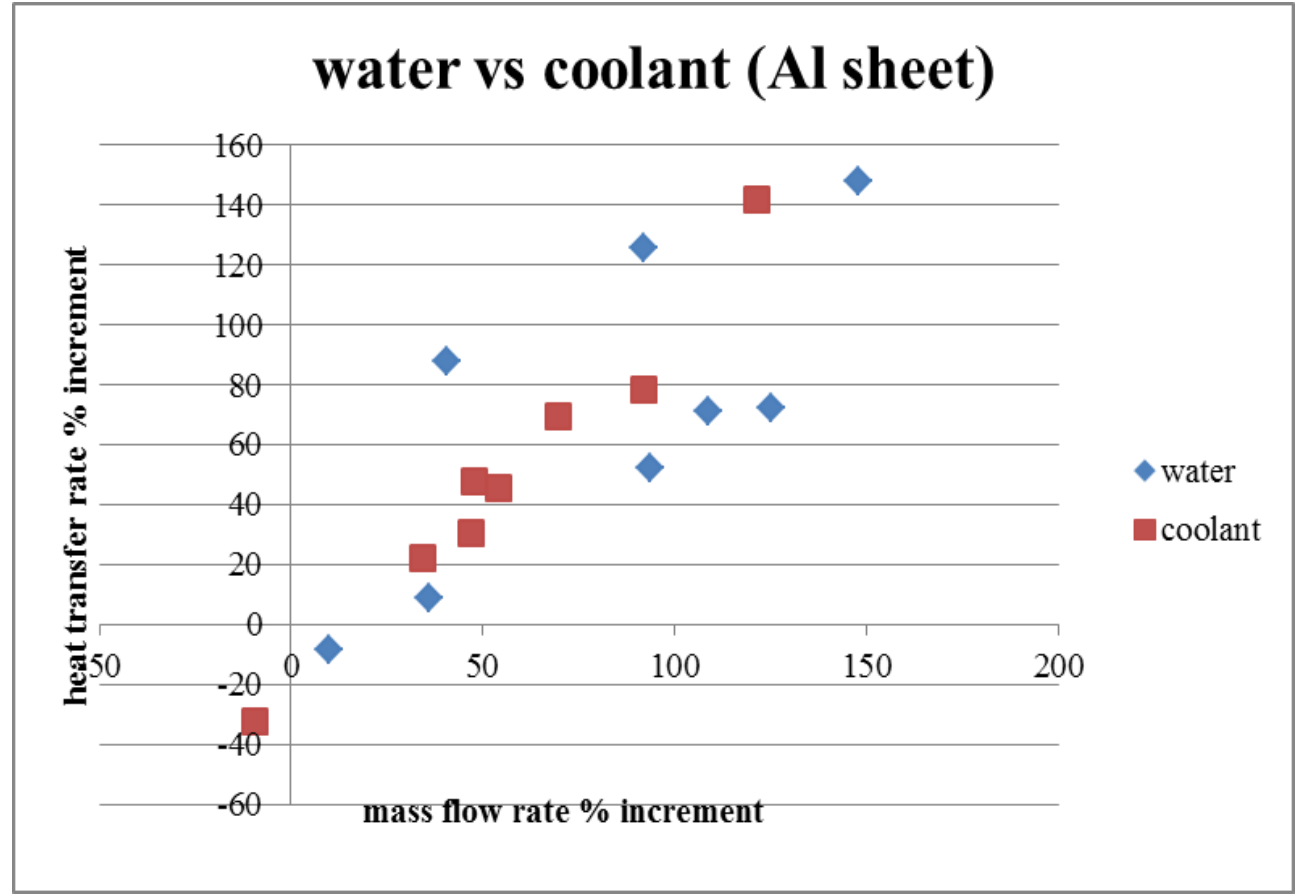

Figure 9. Graph plotted between $\%$ increment of mass flow rate and $\%$ increment in heat transfer rate with various flowing fluids for aluminium sheet collector 


\section{Conclusions}

In this experimental work, lots of measurements have been taken on the two parabolic solar collectors, namely a mirror collector and an aluminium sheet collector. Outlet temperatures, heat transfer rates and instantaneous efficiencies have been recorded with combinations of two water/coolant flowing fluids and four pipe materials and optimized performance of solar collectors are determined. Through the experimentation, maximum heat transfer rate and maximum instantaneous efficiency of the collector have been identified. The conclusion is that the maximum instantaneous efficiency of $92 \%$ is achieved at 12:30PM with aluminium pipe-water combination through aluminium sheet collector. The maximum heat transfer rate of $1208.99 \mathrm{~W}$ is achieved at 01:30PM with copper-antifreeze ethylene glycol through mirror collector. Furthermore, the maximum temperature difference of $12.2^{\circ} \mathrm{C}$ is achieved at $12: 30 \mathrm{PM}$ with copper-water combination though aluminium sheet collector. With the help of graphical analyses, this experimental study concludes copper is the best pipe materials. And, antifreeze ethylene glycol coolant with copper pipe would absorb more heat than water. The reflection of solar energy to the absorber pipe via aluminium sheet collector is better than mirror collector. The conclusions are useful for future improvement in the performance of parabolic solar collectors.

\section{Acknowledgements}

This research work is done at Sushila Devi Bansal College of Technology, Indore, Madhya Pradesh, India. Fabrication of parabolic solar collector is done by research scholar.

\section{REFERENCES}

[1] Sukhatme S. P., Nayak J. K., "Solar Energy Principles of Thermal Collection and Storage", $3{ }^{\text {rd }}$ Edition, The McGraw Hill Companies, New Delhi, India, 2011.

[2] Kumar K. V. P., Srinath T., Reddy V., "Design, Fabrication and Experimental Testing of Solar Parabolic Trough Collectors with Automated Tracking Mechanism", International Journal of Research in Aeronautical and Mechanical Engineering, Volume 01, Issue 04, pp 37-55, 2013.

[3] Tzivanidis C., Bellos B., Korres D., Antonopoulos K. A., Mitsopoulos G., "Thermal and Optical Efficiency Investigation of A Parabolic Trough Collector", Case Studies in Thermal Engineering, Volume 06, pp 226-237, 2015. http://dx.doi.org/10.1016/j.csite.2015.10.005.

[4] Liu X., Huang J., Mao Q., "Sensitive Analysis for the Efficiency of a Parabolic Trough Solar Collector Based on Orthogonal Experiment", International Journal of Photoenergy, Volume 2015, Article ID 151874, pp 1-7, http://dx.doi.org/10.1155/2015/151874.

[5] Mohamad A., Orfi J., Alansary H., "Heat Losses from Parabolic Trough Solar Collectors", International Journal of Energy Research, Volume 38, pp 20-28, 2014. http://dx.doi.org/10.1002/er.3010.

[6] Geete A., Kothari S., Sahu R., Likhar P., Saini A., Singh A., "Experimental Analysis on Fabricated Parabolic Solar Collector with Various Flowing Fluids and Pipe Materials", International Journal of Renewable Energy Research, Volume 06, Issue 04, 2016. 